\title{
DUSEL-related Science at LBNL Program and Opportunities
}

Christian Bauer, Jason Detwiler, Stuart Freedman, Murdock Gilchriese, Richard Kadel, Volker Koch, Yury Kolomensky, Kevin Lesko, Zoltan Ligeti (co-chair), Henrik von der Lippe, Steve Marks, Yasunori Nomura, David Plate, Natalie Roe, Ernst Sichtermann (co-chair)

(DUSEL Experimental Program Study Committee) 


\section{Contents}

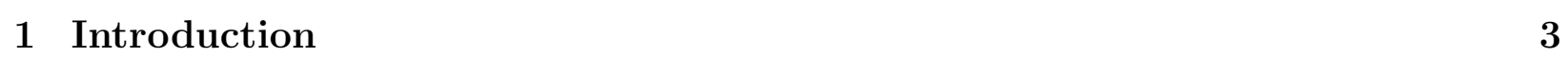

2 Nuclear / Particle Astrophysics $\quad 6$

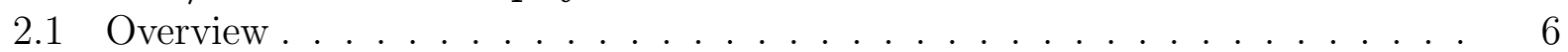

2.2 Ongoing Berkelev activities . . . . . . . . . . . . . . . . 8

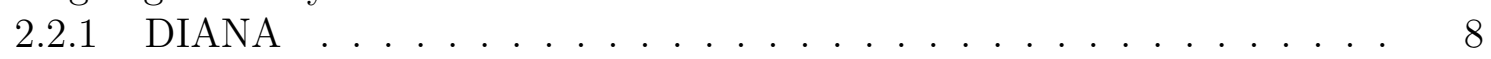

2.2 .2 IceCubs . . . . . . . . . . . . . . . . . . . . . . . . . . . . . . . . . . . . . . . . . . . . .

2.3 Opportunities . . . . . . . . . . . . . . . . . . . . . . 11

$3 \quad$ Neutrinoless double beta decav 12

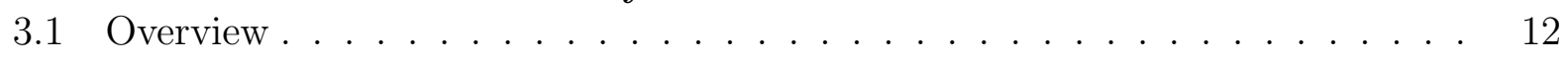

3.2 Ongoing Berkelev activities . . . . . . . . . . . . . . . . . . . 13

3.2 .1 MAJORANA . . . . . . . . . . . . . . . . . . . . . . 13

3.2.2 CUORICINO / CUORE / Future upgrades . . . . . . . . . . . . . 15

3.2.3 High pressure Xenon detector R\&D . . . . . . . . . . . . . . . . 16

3.3 Opportunities . . . . . . . . . . . . . . . . . . 16

\begin{tabular}{|ll}
\hline Neutrino oscillations & 18
\end{tabular}

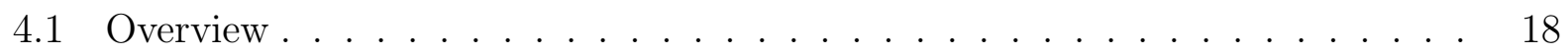

4.2 Ongoing Berkelev activities . . . . . . . . . . . . . . . . . . . . 19

4.2.1 Sudburv Neutrino Observatorv . . . . . . . . . . . . . . . . . . 19

4.2 .2 KamLAND . . . . . . . . . . . . . . . . . . . . . . . . . . . . . . . . . . . . . . . .

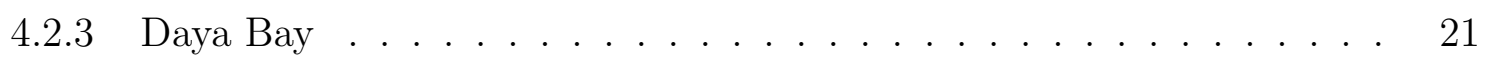

4.2.4 Long baseline neutrino experiment . . . . . . . . . . . . . . . . . . . . 21

4.3 Opportunities . . . . . . . . . . . . . . . . . . . . . . . 22

5 Dark matter searches 23

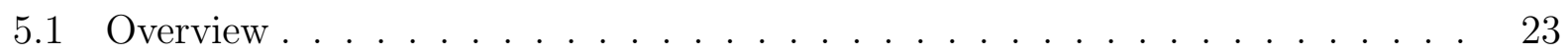

5.2 Ongoing Berkelev activities . . . . . . . . . . . . . . . . . . . . 24

5.2.1 CDMS / Super-CDMS / GEODM . . . . . . . . . . . . . . . . . 24

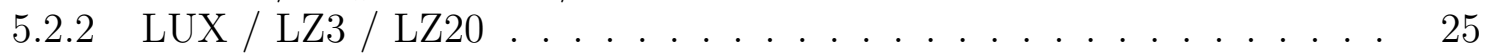

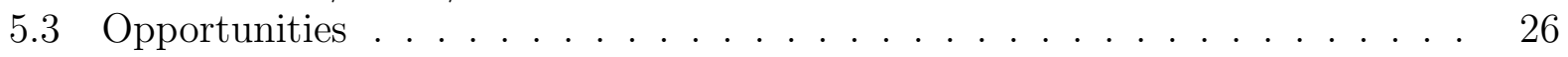

6 Recommendations 26

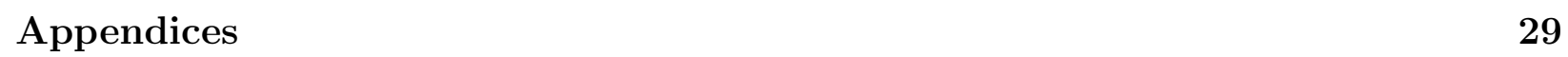

A Low Background Counting and Radiological Assay . . . . . . . . . . . . . . 29

B DUSEL Working Group . . . . . . . . . . . . . . . . . 29 


\section{Introduction}

The National Science Foundation is advancing the design of a Deep Underground Science and Engineering Laboratory (DUSEL) at the former Homestake mine in South Dakota. UC Berkeley and LBNL are leading the design effort for the facility and coordinating the definition and integration of the suite of experiments to be coupled to the facility design in the creation of an MREFC (Major Research Equipment and Facility Construction) proposal. The State of South Dakota has marshaled $\$ 120 \mathrm{M}$ to prepare the site and begin a modest science program at the $4850 \mathrm{ft}$ level. The first physics experiment is anticipated to begin installation in 2009.

The current timetable calls for the MREFC Preliminary Design to be assembled by 2010 to be presented to the National Science Board in 2011. This, in turn, indicates that the earliest DUSEL construction start would be FY2013. The MREFC is estimated (before the inclusion of the long baseline neutrino components) at $\$ 500-600 \mathrm{M}$, roughly divided evenly between the experimental program and support for the facility. Construction was estimated at 6-8 years. The DOE and NSF are establishing a Joint Oversight Group (JOG) to coordinate the experimental programs and participation in DUSEL. It is anticipated that the JOG would mirror the similar function for the NSF and DOE participation in the LHC, and that DOE-HEP, DOE-NP, and NSF will all participate in the JOG.

In parallel with the NSF efforts, DOE-HEP plans to develop a long baseline neutrino program with neutrino beams created at FNAL and aimed at DUSEL. In the P5 report the focus of the program is to pursue $C P$ violation in the lepton sector. The same detectors can also be used for nucleon decay experiments. DOE has indicated that FNAL would be the "lead lab" for the long baseline neutrino program and be charged with designing and implementing the neutrino beamline. BNL is to be charged with designing and implementing the detector. The P5 report also emphasizes the importance of dark matter and neutrinoless double beta decay searches.

The Nuclear Physics Long Range Plan strongly endorses DUSEL and the associated nuclear physics programs. It mentions, in particular, neutrinoless double beta decay, and accelerator-based nuclear astrophysics measurements as key elements of the DUSEL nuclear physics experimental program.

There are numerous fundamental scientific questions that experiments which can naturally be sited at DUSEL can address. LBNL has a long tradition and track record of successful experiments in all of these areas: neutrino physics, dark matter searches, and nuclear astrophysics. Clearly, DUSEL presents many scientific opportunities, and the committee was charged to present a roadmap for LBNL participation, the impact that LBNL is likely to have on experiments at the present level of effort, the value of additional manpower, and opportunities for synergistic Detector R\&D activities.

The Berkeley community is already deeply involved in a number of experiments and/or proposals, shown in Table 1, that will be relevant to science at DUSEL. The approximate time lines for all projects considered in this report are shown in Table 2, For the DUSELrelated experiments the depth at which they would be located is also shown.

Section 2 of this report deals with nuclear astrophysics. Section 3 discusses neutrinoless 


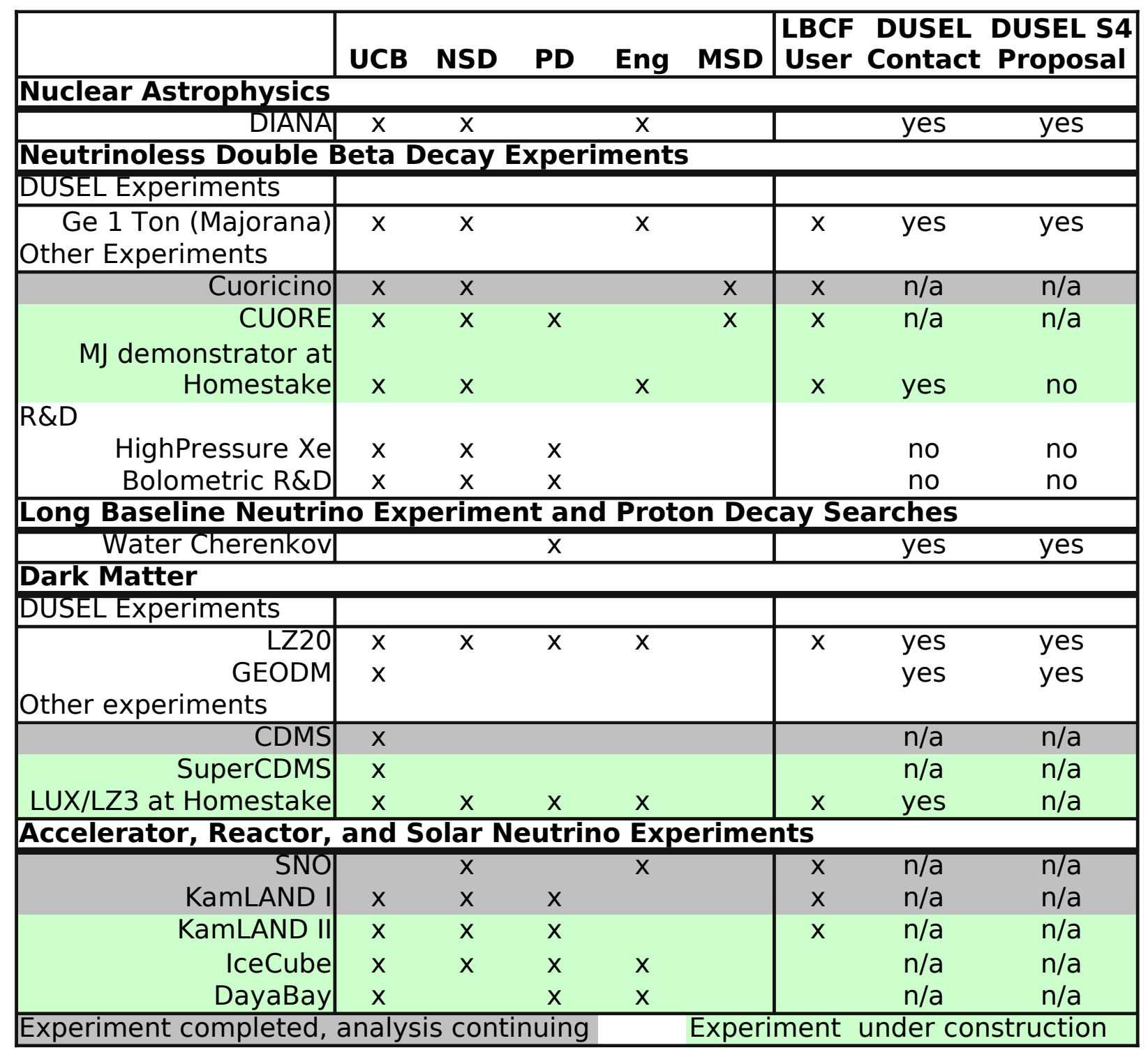

Table 1: Current and imminent Berkeley involvement in DUSEL-related experiments. In some cases the PD and UCB, or the NSD and UCB check marks refer to the same individuals. Note that some of the check marks correspond to substantial, some to modest involvements. The low background counting facility (LBCF) is described in Appendix $\mathrm{A}$.

double beta decays. Section 4 focuses on neutrino oscillations, including the search for $C P$ violation and proton decay. Section 5 deals with dark matter searches. In each section we give a brief overview of that field, review the present Berkeley efforts, and discuss the opportunities going into the future. Section [ 6 contains our recommendations. 


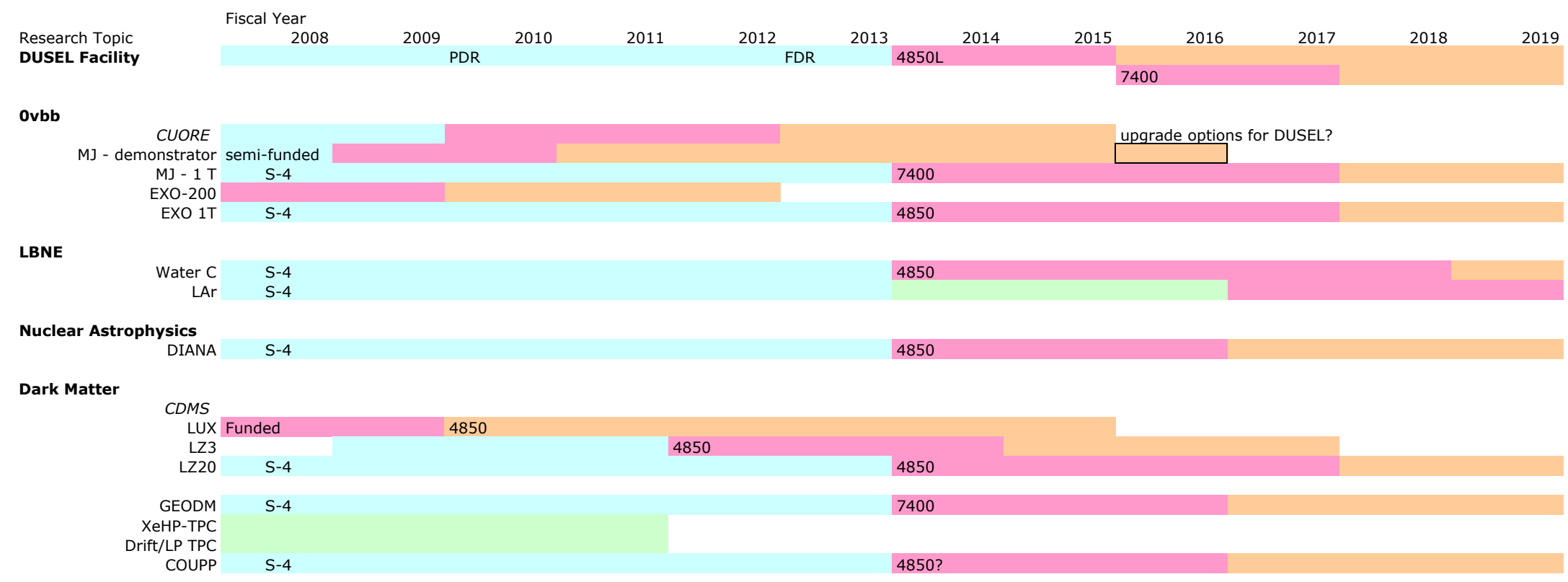

Theta13

Daya Bay

Solar Neutrinos

SNO
KamLAND

Analysis Only upgrade

Neutrino Astronomy

ICECUBE

R\&D
Proposal

Proposal

Construction
Operation

Table 2: Approximate timelines of projects considered. 


\section{Nuclear / Particle Astrophysics}

\section{$2.1 \quad$ Overview}

Nuclear Astrophysics is concerned with the origin of elements in stars and stellar explosions through charged particle, neutron, and weak interaction induced nuclear processes. The field, at the intersection of nuclear physics, astrophysics, and observational astronomy, explores the origin of the elements in our universe and identifies the observational signatures. Experimental Nuclear Astrophysics is characterized by four major directions, nucleosynthesis processes in stars, which is studied with low energy accelerator experiments, explosive nucleosynthesis processes, which requires measurements far from stability with radioactive beams, neutron-induced nucleosynthesis in late stellar evolution, which is pursued at reactor and neutron spallation facilities, and finally neutrino-induced nucleosynthesis processes, which is still largely confined to theoretical prediction and observation. Of these, realistically only the low energy accelerator experiments are relevant for this report which discusses opportunities in underground science. Thus, the science topics of interest are stellar nucleosynthesis and its implications for neutrino measurements.

Stellar nucleosynthesis in the first star generations represents the onset of galactic chemical evolution and prepares the seed for stellar explosion mechanisms. Stellar nucleosynthesis provides the seed and the fuel for explosive nucleosynthesis from type II core collapse supernovae to type Ia thermonuclear supernovae. Current stellar model simulations are at a level of precision that the nuclear reaction rates represent a major source of uncertainty for theoretical predictions and for the analysis of observational signatures. Since the typical thermal energies in stellar environments are below the Coulomb barrier, the reaction cross sections are extremely small, requiring the reduced backgrounds of an underground site for their precise determination. Thus, in order to make further progress, low energy, high intensity underground accelerator facilities are needed. This will significantly improve the understanding of the following science topics associated with low energy stellar burning:

- Solar neutrino sources and the metallicity of the Sun: Low energy nuclear reactions in stellar burning play a critical role for the understanding of the origin of the elements and the processes taking place in the interiors of stars. Proton capture reactions drive stellar hydrogen burning through the pp-chains in low mass stars like our Sun, while proton capture reactions in the $\mathrm{CNO}$ cycles and the NeNa cycles dominate hydrogen burning in massive stars. These reactions are not only responsible for the energy generation and timescale of the main sequence phase of stellar burning but also generate the neutrinos that can be used as direct probes for observing nuclear burning in the core of our Sun. The experimental uncertainties in the nuclear reaction rates associated with these processes translate directly into the uncertainties for the predicted neutrino production from our Sun and other stellar burning scenarios. With high precision measurements of the neutrino production rates coupled with the observation of the solar neutrino flux cross sections, we can directly determine the metallicity of the solar core. The key reactions in this context are ${ }^{3} \mathrm{He}(\alpha, \gamma){ }^{7} \mathrm{Be}$ which determines the branch between the first and second pp-chain and therefore the ratio of pp-neutrinos versus 
the high energy ${ }^{8} \mathrm{Be}$ neutrinos from the third pp-chain. This reaction together with the ${ }^{7} \mathrm{Be}(\mathrm{p}, \gamma)$ reaction, are the essential remaining uncertainties in pp-chain measurements.

- Carbon-based nucleosynthesis: Alpha capture reactions characterize stellar helium burning in Red Giant (RG) stars and Asymptotic Giant Branch (AGB) stars. These processes play a key role in stellar nucleosynthesis of first and early star generations where carbon is formed first by the triple alpha process on primordial helium. Particularly critical are reactions such as ${ }^{12} \mathrm{C}(\alpha, \gamma){ }^{16} \mathrm{O}$ and ${ }^{16} \mathrm{O}(\alpha, \gamma){ }^{20} \mathrm{Ne}$ that define the ${ }^{12} \mathrm{C} /{ }^{16} \mathrm{O}$ abundance ratio in the subsequent carbon burning stage that is driven by fusion processes such as ${ }^{12} \mathrm{C}+{ }^{12} \mathrm{C}$ and ${ }^{16} \mathrm{O}+{ }^{12} \mathrm{C}$. The interplay of these reactions influence the carbon-oxygen distribution in the post helium burning or post carbon burning matter, dictate the light element abundance distribution in White Dwarf matter and, therefore, define the ignition and burning conditions for nova explosions as well as type Ia supernova explosions. The latter is of particular relevance for modeling type Ia supernovae reliably and interpreting their empirical role as standard candles in cosmology studies.

- Neutron sources for the production of trans-Fe elements in stars: The s-process nucleosynthesis of heavy elements in stars depends on the neutron flux in stars. The reactions ${ }^{13} \mathrm{C}(\alpha, \mathrm{n}),{ }^{17} \mathrm{O}(\alpha, \mathrm{n})$, and ${ }^{22} \mathrm{Ne}(\alpha, \mathrm{n})$ are considered the most likely sources for neutron production in a variety of stellar helium burning and carbon burning environments. The low energy cross sections of these reactions define the neutron flux at the various burning conditions and therefore determine the production of s-process elements in core helium burning and shell carbon burning of massive stars as well as in intershell helium burning region of AGB stars.

\section{Present Experimental Status and Limitations}

Nuclear reaction rates in stars are very small because the typical thermal energies are considerably below the Coulomb barrier. In addition, due to the interplay between the thermal distribution and the Coulomb barrier, nuclear reactions inside a star happen within a small energy window, the so called Gamow window. Therefore, laboratory experiments need to measure the relevant nuclear cross sections in or close to the Gamow window, where the rates are exceedingly small. Extrapolations of experimental results to the relevant energy regions are inherently uncertain as they involve detailed assumptions about the reaction mechanisms or resonance structures. These uncertainties often span many orders of magnitude and translate into substantial uncertainties for nucleosynthesis simulations as well as simulations of stellar evolution scenarios. So far few reactions have been directly measured in the relevant solar energy range, namely the pp-chain reaction ${ }^{3} \mathrm{He}\left({ }^{3} \mathrm{He}, 2 \mathrm{p}\right){ }^{4} \mathrm{He}$ and the CNO cycle reaction ${ }^{14} \mathrm{~N}(\mathrm{p}, \gamma)^{15} \mathrm{O}$, the slowest reaction in the CNO cycles that determines the overall energy and neutrino production rate associated with stellar CNO burning. Both have been measured at the European Laboratory for Underground Nuclear Astrophysics (LUNA) in the Gran Sasso underground laboratory, which is presently the only operating underground facility in the world for these types of experiments. There are initiatives for 
future underground accelerator laboratories in India, Romania, Spain, and in the United Kingdom. An upgrade for LUNA is also being discussed in Italy since the facility has been extremely successful and has convincingly demonstrated the importance and the advantages of underground accelerator experiments. The present LUNA facility is small and is limited to the measurement of proton capture reactions below $400 \mathrm{keV}$ with typical beam currents between 100 and $200 \mu \mathrm{A}$. This is sufficient for capture measurements at low Z target nuclei. Higher beam currents are necessary to expand these measurements to lower energies with sufficient statistics. Higher energies are necessary to expand the physics program into the higher $\mathrm{Z}$ range, which is important for stellar burning in massive stars and even in explosive Mg-Al burning in novae. Alpha capture measurements require substantially higher energies than available at LUNA. In addition, many reactions have complex resonance structures which require mapping out the cross sections over a wide range in order to make reliable extrapolations to lower energies.

The experimental difficulties in determining the low energy cross sections are caused by large background rates associated with cosmic ray-induced reactions, background from natural radioactivity in the laboratory environment, and the beam-induced background on target impurities. Natural background can be reduced by careful shielding of the target and detector environment, and beam-induced background can be reduced by active shielding techniques through event identification, but it is difficult to reduce the background component from cosmic ray muons. An underground location reduces the cosmic ray-induced background by several orders of magnitude, allowing measurements to be pushed to far lower energies than presently possible. This has been clearly demonstrated at LUNA by the successful studies of critical reactions in the pp-chains and first reaction studies in the CNO cycles.

\subsection{Ongoing Berkeley activities}

\subsubsection{DIANA}

Daniela Leitner and Paul Vetter of NSD and Matthaeus Leitner from ED have conceived and developed the concept of a low energy nuclear astrophysics facility, the Dakota Ion Accelerators for Nuclear Astrophysics (DIANA), to be sited at the $4850 \mathrm{ft}$ mid-level campus in DUSEL. Using LDRD support they developed the basic design of the low energy accelerator and a concept for the high energy accelerator. One of the key elements of the LDRD was to mobilize the low energy astrophysics community to establish a next generation underground facility at the DUSEL laboratory. Letters of Intent were submitted to the DUSEL Homestake site and a formal collaboration was formed. Michael Wiescher and Daniela Leitner act as working group leaders for the nuclear astrophysics underground accelerator group and were instrumental in producing a community supported white paper at the DUSEL Town meeting in Washington in November of 2007. This white paper establishes the scientific case and includes preliminary parameters for the proposed facility. In January of 2009, the collaboration submitted a joined S4 proposal to NSF to develop a Preliminary Design Report for the DIANA facility at the $4850 \mathrm{ft}$ mid-level campus. At present the collaboration consists of the Institute for Structure and Nuclear Astrophysics at Notre Dame, 
the Nuclear Astrophysics Group at Univ. of North Carolina, the Physics Department at Western Michigan Univ., the Colorado School of Mines, and the Nuclear Science Division and the Engineering Division at LBNL. In addition to the core institutes there are connections via the Joint Institute of Nuclear Astrophysics (JINA) with Yale University, Indiana University, Ohio University, University of Washington, Horia Hulibei National Institute for Nuclear Physics and Nuclear Engineering (HH-NIPNE) Romania, National Institute of Nuclear Physics (INFN) Genova, Italy, and INFN Naples, Italy, which are also part of the underground science working group. The S4 DIANA proposal is led by Notre Dame, with Wiescher as principal investigator and project director. Daniela Leitner (NSD) is the project physicist and Matthaeus Leitner (ED) is in charge of project management.

The basic concept of DIANA consists of two accelerators: a low energy $400 \mathrm{kV}$ high intensity accelerator and a second accelerator with a maximum voltage of $3 \mathrm{MV}$, together with the necessary target developments and detector systems. The low energy accelerator will complement the present activities at LUNA and improve the experimental conditions. In particular, it will address the question of solar neutrino production to determine the metallicity of the solar core. The second accelerator with a maximum voltage of $3 \mathrm{MV}$ is needed to address nucleosynthesis problems in stellar helium and carbon burning. In particular, it will address carbon-based nucleosynthesis and the neutron production for s-process nucleosynthesis at these sites. This 3 MV machine extends well beyond existing machines in the field and is not feasible at the confined space conditions of the existing caverns at the Gran Sasso laboratory. Progress in low energy stellar cross section measurements depends critically on the development of new target and detector technology. The DIANA proposal, therefore, calls for the development of a new (segmented) gamma detector array that provides active shielding possibility through event identification and background rejection. Other detectors will include silicon detector arrays and high efficiency thermalized neutron detector systems.

The proposed DIANA facility will improve the experimental reach for all three key topics outlined above:

- Solar Neutrino Sources and the Metallicity of the Sun

The new design for the $400 \mathrm{keV}$ accelerator developed by the LBNL accelerator and ion source group is expected to provide high-intensity beams at low energies well in excess of $1 \mathrm{~mA}$. This will allow for the measurement of the critical reactions in stellar hydrogen burning, ${ }^{3} \mathrm{He}(\alpha, \gamma){ }^{7} \mathrm{Be}$ and ${ }^{7} \mathrm{Be}(\mathrm{p}, \gamma)$, in or near the Gamow window. The new facility will also enable the measurement of key CNO cycle reactions, such as ${ }^{14} \mathrm{~N}(\mathrm{p}, \gamma){ }^{15} \mathrm{O}$, ${ }^{12} \mathrm{C}(\mathrm{p}, \gamma){ }^{13} \mathrm{~N},{ }^{15} \mathrm{~N}(\mathrm{p}, \gamma){ }^{16} \mathrm{O},{ }^{17} \mathrm{O}(\mathrm{p}, \gamma){ }^{18} \mathrm{~F}$, and ${ }^{15} \mathrm{~N}(\mathrm{p}, \alpha){ }^{12} \mathrm{C}$, within $50 \mathrm{keV}$ of the Gamow window. This will reduce the uncertainty in CNO neutrino production in our Sun and will, together with precise ${ }^{8} \mathrm{Be}$ and $\mathrm{CNO}$ neutrino identification, constrain the determination the primordial solar metallicity, which is a critical parameter for Standard Solar Model predictions. A recent study indicates a substantially lower metallicity causing a disagreement with the solar model predictions based on helioseismology.

- Carbon-based Nucleosynthesis

The key reactions for carbon-based nucleosynthesis, ${ }^{12} \mathrm{C}(\alpha, \gamma){ }^{16} \mathrm{O}$ and ${ }^{16} \mathrm{O}(\alpha, \gamma){ }^{20} \mathrm{Ne}$, at present are only measured in above ground experiments and are limited to energies 
above $0.9 \mathrm{MeV}$ and $1.1 \mathrm{MeV}$. With the proposed $3 \mathrm{MV}$ machine together with improved target technology, it should be possible to extend the measurement of ${ }^{12} \mathrm{C}(\alpha, \gamma){ }^{16} \mathrm{O}$ down to a center-of-mass energy below $700 \mathrm{keV}$ and to consistently measure the cross section up to $4.5 \mathrm{MeV}$. This will reduce the uncertainties of the extrapolation into the stellar energy range near $300 \mathrm{keV}$, which are due to broad resonance structures direct capture contributions, and multiple interference patterns.

- Neutron Sources for the Production of Trans-Fe Elements in Stars The two reactions considered to be the main sources of stellar neutrons ${ }^{13} \mathrm{C}(\alpha, \mathrm{n})$ and ${ }^{22} \mathrm{Ne}(\alpha, \mathrm{n})$ as well as the secondary sources, ${ }^{17} \mathrm{O}(\alpha, \mathrm{n})$ and ${ }^{25} \mathrm{Mg}(\alpha, \mathrm{n})$, can be measured with the new facility. While the reaction components in the ${ }^{13} \mathrm{C}(\alpha, \mathrm{n}){ }^{16} \mathrm{O}$ neutron source recently have been studied and evaluated over a wide energy range, a reliable extrapolation of the low energy sub-threshold resonance component requires the measurement at substantially lower energies, which should be feasible with the low energy $400 \mathrm{kV}$ machine. In addition the $3 \mathrm{MV}$ machine will allow high energy measurements necessary to identify the strength and pattern of the various complex reaction components over a wide energy range.

At present very little is known about the low energy cross sections for the second neutron source, ${ }^{22} \mathrm{Ne}(\alpha, \mathrm{n})^{25} \mathrm{Mg}$. The competing channel ${ }^{22} \mathrm{Ne}(\alpha, \gamma){ }^{26} \mathrm{Mg}$ should also be measured. Both these measurements are essential to reduce one of the main uncertainties in nucleosynthesis simulations of massive stars and AGB stars and are part of the initial suit of experiments of the DIANA proposal.

\subsubsection{IceCube 1}

IceCube is a $1 \mathrm{~km}^{3}$ neutrino observatory being built at the South Pole. The main physics goal of IceCube is to search for high-energy $(10 \mathrm{GeV}$ to $1 \mathrm{EeV})$ neutrinos from extra-terrestrial sources, and thereby determine the source of the high-energy cosmic rays.

In addition to this main goal, IceCube has secondary physics goals of which some have overlap with the other physics areas discussed in this report. One key IceCube topic is the search for neutrinos produced by Weakly Interacting Massive Particle (WIMP) annihilation in the Earth or the Sun. WIMPS are heavy, and, over time, are gravitationally captured in bodies like the Earth and the Sun. If the WIMP density is high enough, they will selfannihilate (in most models, they are Majorana particles), producing neutrinos which may be detected by IceCube. IceCube has published limits on neutrinos from the Sun. Because the Sun is mostly hydrogen, this analysis is very sensitive to the spin-dependent neutralinoproton cross section, and the limits are orders of magnitude smaller than the limits from direct experiments. Future limits should give competitive direct constraints on SUSY models, even accounting for expected improvements by other experiments.

IceCube is also studying neutrino oscillations. One analysis looks for non-standard models of neutrino oscillations, such as are expected from phenomena like additional dimensions or modifications to Lorentz invariance. A similar analysis of AMANDA-II data has been

\footnotetext{
${ }^{1}$ We thank Spencer Klein for help with writing this section.
} 
published. A second study will use the DeepCore infill array (construction has started; will be complete by next year) to lower IceCube's threshold to well below $100 \mathrm{GeV}$, and look for $\nu_{\mu}$ disappearance and $\nu_{\tau}$ appearance due to conventional oscillations. If $\sin 2 \theta_{13}$ is not small (near the current CHOOZ limit) and systematic effects can be adequately controlled, then DeepCore may be able to study the neutrino mass hierarchy.

The IceCube effort at LBNL began in 1994, with the conception and prototyping of digital optical modules using LBNL's electronics core competence. IceCube construction began in 2005, and should be complete by 2011. LBNL designed, built, and tested over 5900 DOM main boards; these are the complete data acquisition hardware for the optical modules. LBNL also had major responsibilities for the DOM firmware, and the surface hardware and overall DAQ and experiment control software. Much of the off-line calibration and simulation software was developed at LBNL. LBNL has a lead role in several analyses of data from the partial detector. The aim is to collect data with the completed array for $\sim 10$ years, with data analysis continuing during that time. The LBNL group, currently led by Klein (NSD \& UCB), includes Nygren (PD), Goldschmidt (NSD), Kiryluk (NSD \& UCB), with significant engineering (Stezelberger, Eng.) and computing (Beattie, CRD) support, and two postdocs and students.

For the future, the group is pursuing ARIANNA, a proposed $100 \mathrm{~km}^{3}$ detector on the Ross Ice Shelf. ARIANNA will look for radio waves from showers produced by ultra-high energy (UHE, above $10^{17} \mathrm{eV}$ ) neutrinos. These neutrinos come from GZK interactions of cosmic-ray protons with the cosmic microwave background; ARIANNA will study GZK neutrinos from sources at cosmological distances (redshifts out to 2-3) and thereby probe the high-energy history of the universe. ARIANNA will also look for more speculative sources of neutrinos, including nearby point sources. It will also measure the neutrino-nucleon cross section at very high energies, and may also use these UHE neutrinos for other studies of fundamental physics, such as non-standard oscillations that appear at very high energies. The LBNL group is working on prototype electronics for ARIANNA, and plans to play a key role in ARIANNA hardware.

\subsection{Opportunities}

The DIANA facility at DUSEL presents an obvious opportunity for LBNL, specifically NSD, to seriously enter the field of nuclear astrophysics. With the arrival of Wick Haxton this is especially timely. In addition, as described above, the experiments carried out with DIANA have a direct bearing on precision solar neutrino measurements, and thus nicely complement the already strong neutrino program at LBNL. At present LBNL's involvement in DIANA is mostly on the accelerator side. However, for a serious involvement of NSD in this field to be sensible, one would have to build a sufficiently strong experimental research program which will be able to harvest the investment into the facility. To accomplish the goal of scientific involvement in this facility, it will be crucial to establish interest and support from DOE for this program. Michael Wiescher has presented the DIANA proposal to the DOE Low Energy Nuclear Science program managers and NSD should further establish the scientific case with DOE. There is a clear opportunity with the DUSEL project, since the NSF and 
DOE are presently actively negotiating an agreement.

NSD should consider (re)allocating resources in order to build an experimental nuclear astrophysics program. In view of the arrival of Wick Haxton at Berkeley, such a development appears rather sensible. In addition, during the construction and commissioning phase, astrophysics cross section measurements should be carried out, above ground, at LBNL. This would ensure that the DIANA components meet specifications, build up local expertise, and would allow starting a physics program. One could imagine that some complementary measurements as well as detector development could be carried out the 88-Inch Cyclotron.

The DIANA S4 proposal specifically calls for the development of a new generation of (segmented) gamma detectors. Given the leading expertise by NSD in gamma detection developments, this could present another opportunity for NSD involvement in this project.

\section{$3 \quad$ Neutrinoless double beta decay}

\section{$3.1 \quad$ Overview}

Over the last decade tremendous progress has been made toward understanding neutrino properties. Nonzero neutrino masses have been established through neutrino oscillations, and two of the three mixing angles, $\theta_{12}$ and $\theta_{23}$, have been measured (see Sec. 4.11).

A crucial question is whether neutrinos are Majorana or Dirac type fermions. In the case of Dirac neutrinos, we introduce new degrees freedom to the standard model, namely right-handed neutrinos, which do not have charges under any of the standard model gauge groups. Lepton number is conserved in this case. On the other hand, in the case of Majorana neutrinos, we do not introduce any new light degrees of freedom, but rather identify righthanded neutrinos with anti-neutrinos, which violates lepton number. In this case, the see-saw mechanism can explain the small neutrino masses, as resulting from the mixing between the light left-handed and very heavy right-handed new "neutrino" states, as $m_{\nu} \sim m_{D}^{2} / M \ll v$. Here $m_{D}$ is a typical order of magnitude of quark and lepton masses, $M$ is the mass of the right-handed heavy "neutrinos", and $v \sim 250 \mathrm{GeV}$ is the electroweak scale. The atmospheric neutrino mass scale for the third generation, combined with a naive estimate $m_{D} \sim m_{t}$ gives $M \sim 10^{15} \mathrm{GeV}$, tantalizingly close to the energy scale where the gauge coupling constants unify in the minimal supersymmetric standard model. While theorists' bias has (mostly) been in favor of Majorana neutrinos, we will not know what is the correct extension of the Standard Model to incorporate the finite mass of neutrinos unless we know whether the neutrino mass is of Dirac or Majorana type.

Currently the only promising approach to discriminate Dirac and Majorana neutrinos is neutrinoless double beta decay $(0 \nu \beta \beta)$ experiments. They look for lepton number violation in the decay of heavy nuclei, $(A, Z) \rightarrow(A, Z+2)+2 e^{-}$, with no accompanying electron anti-neutrinos. The rate for neutrinoless double $\beta$ decay can be written as

$$
\left[T_{1 / 2}^{0 \nu}\right]^{-1}=G^{0 \nu}\left|M^{0 \nu}\right|^{2}\left\langle m_{\beta \beta}\right\rangle^{2}, \quad\left\langle m_{\beta \beta}\right\rangle=\left|\Sigma_{i=1}^{3} m_{i} U_{e i}^{2}\right|
$$

where $G^{0 \nu}$ is a calculable phase space factor, $M^{0 \nu}$ is the matrix element for the transition 
(typically known to about a factor of two). The "effective neutrino mass", $\left\langle m_{\beta \beta}\right\rangle$, depends on the masses, mixing angles, and $C P$ violating phases in the mixing matrix [see Eq. (2)].

So far we do not know the absolute masses of the neutrinos, so $\left\langle m_{\beta \beta}\right\rangle$ has large uncertainties. Only mass squared differences have been measured in oscillation experiments (see Sec. 4.1) and an upper bound on the sum of masses has been set from cosmology, $\sum m_{i} \lesssim 1 \mathrm{eV}$. The results of oscillation experiments suggest a minimum neutrino mass that the next generation of $0 \nu \beta \beta$ experiments may be able to probe. If the mass spectrum is the inverted hierarchy, which the long baseline experiments can demonstrate if true, the two neutrino states with the smaller (solar) mass splitting have masses at least the square root of the larger (atmospheric) mass splitting: $m_{1}, m_{2}>\left(\Delta m_{23}^{2}\right)^{1 / 2} \gtrsim 0.05 \mathrm{eV}$. Even if $U_{e 1}^{2}$ and $U_{e 2}^{2}$ tend to cancel, they cannot completely do so, because $\theta_{12}$ is not maximal. Therefore, in case of inverted hierarchy, a sensitivity down to about $0.01 \mathrm{eV}$ would discover or exclude that neutrinos are Majorana. On the other hand, if the mass spectrum is the normal hierarchy, $\left\langle m_{\beta \beta}\right\rangle$ can be very small, and in this case it is possible that none of the proposed experiments would be conclusive.

Crucial to this type of experiment is an excellent energy resolution, $\Delta E / E \lesssim 0.1 \%$, to distinguish $0 \nu \beta \beta$ from the continuous spectra of allowed second order $2 \nu \beta \beta$ decay. Many choices of nuclei and detection methods have been suggested. So far only the HeidelbergMoscow experiment, using Ge detectors, has reported a positive signal, corresponding to $\left\langle m_{\beta \beta}\right\rangle \sim(0.4-1.3) \mathrm{eV}$.

\subsection{Ongoing Berkeley activities}

\subsubsection{MAJORANA}

The MAJORAnA Experiment aims to perform a search for $0 \nu \beta \beta$ decay in ${ }^{76}$ Ge using modular arrays of enriched high-purity Germanium (HPGe) detectors. MAJORANA's approach is to implement advanced HPGe technologies, such as P-type Point Contact (PPC) detectors, in low-mass supports made using ultra-low background materials and techniques. The detector arrays will be arranged in a "standard" vacuum cryostat surrounded by graded passive and active shielding. A European effort, GERDA, is also in the process of fielding several tensof-kg of HPGe detectors in a novel approach in which the detectors are immersed directly in a bath of liquid cryogen. The two collaborations have signed a Letter of Intent to join together for a future ton-scale experiment employing the best technologies and techniques demonstrated in the current R\&D phase. The goal of the ton-scale experiment is to cover, to the extent possible, the inverted hierarchy region for the effective $\left\langle m_{\beta \beta}\right\rangle$ neutrino mass. This experiment will also allow investigation of other double-beta decay physics, for example via high-statistics $2 \nu \beta \beta$ spectral measurements and searches for decays to excited states of the final-state nucleus.

In the short term (the next $\sim 5$ years), the MAJORAnA Collaboration intends to field several tens-of-kg of detector mass at the 4850 level of Sanford Laboratory in an R\&D effort called the Majorana Demonstrator. The primary goals of the Demonstrator are to confirm or refute Klapdor-Kleingrothaus et al.'s claimed observation of $0 \nu \beta \beta$ decay, and to 
demonstrate backgrounds low enough to justify scaling up to the larger ton-scale experiment. By deploying low-threshold PPC detectors, the Demonstrator may be able to explore a number of other physics topics, including dark matter, axions, coherent neutrino-nucleon scattering, and electron decay.

The Majorana Collaboration is currently acquiring $18 \mathrm{~kg}$ of natural-Ge PPC-like detectors and is advancing its detector mount and readout design to maturity by leveraging a combination of DOE R\&D funding (\$250k in 2007 and $\$ 500 \mathrm{k}$ in 2008 ), redirect (especially from SNO) and aligned funding, and institutional support, including significant contributions from national lab LDRD and university startup funds (Wilkerson). The DOE has indicated its intent to begin fully funding the MAJORANA DEMONSTRATOR in FY10 pending a CD1-like review this fall. The collaboration expects to begin construction in FY10 of the first module in the Demonstrator's phased deployment, with physics results starting as early as 2011. All Demonstrator modules are expected to be running by 2013-2014. Construction for a ton-scale experiment could commence in 2015 at the earliest, although more likely a year or two later, lasting 3-5 years. The physics program of the ton-scale experiment would last a decade or more. The Majorana Collaboration submitted an S4 proposal for such a ton-scale experiment at DUSEL.

LBNL has been participating in MAJORANA since $\sim 2003$. During this period, LBNL has had significant leadership roles in detectors, DAQ, simulations and analysis, and materials and assay tasks. Its key contribution to MAJORANA was highly-segmented N-type detector technology. The effort was initially led by Lesko (NSD), who has since become leader of the DUSEL Project Team. Current NSD staff involvement consists in Vetter (NSD, UCB Nucl. Eng.), Chan, Poon, Fujikawa, 3 postdoctoral fellows, and 1 GSRA. Engineering involvement includes Luke, Amman, Zimmermann, and Yaver. LBNL staff presently lead the Detector as well as the Simulations and Analysis tasks.

In the last year, the MAJORANA collaboration has transitioned from highly-segmented N-type detector technology to a plan which favors PPC detectors. This has somewhat diminished LBNL's role in MAJORANA, despite the fact that the early development of PPCs was done at LBNL by Luke in the 1980's, and despite LBNL's ongoing roles in the planning, design, and prototyping of these detectors for the DEMONSTRATOR. LBNL's present contributions to the experiment are focused on the signal readout chain: the detector contact, front-end resistive feedback circuit, pre-amplification, and digitization electronics. LBNL contributes to detector prototyping and design, underground detector fabrication, cabling and connector design, detector mount design, low-background counting and other materials assay (e.g., NAA), detector segmentation and characterization R\&D, facilities design, and DAQ software development. MAJORANA makes significant use of Smith's Low Background Counting Facility. LBNL continues to make major contributions also to simulations and analysis via software development, MC production runs at PDSF, background model development, and R\&D detector characterization and data analysis.

In addition, it should be noted that several other National Laboratories have major roles and involvement in MAJORANA. Early leadership in the experiment arose out of PNNL, whose core competency in electroformed $\mathrm{Cu}$ production and assay are essential to MAJORANA's success. PNNL also leads the module / cryostat / crystal mount design efforts. 
LANL is providing significant LDRD funding for a first module of $18 \mathrm{~kg}$ of PPC-like Canberra "BEGe" detectors. LANL collaborators also contribute significantly to the facilities, shield, and module design, cryostat prototyping and testing, detector calibration and characterization, and background model development. The current Spokesperson is also at LANL (Elliott). ORNL is planning to handle the production of detector-grade enriched $\mathrm{Ge}$, and also contributes significantly to detector design/production/ characterization and data analysis. Both PNNL and ORNL have expressed interest in being the lead lab for the experiment.

\subsubsection{CUORICINO / CUORE / Future upgrades}

CUORE (Cryogenic Underground Observatory for Rare Events) is a cryogenic-bolometer detector consisting of $988 \mathrm{TeO}_{2}$ crystals, $750 \mathrm{~g}$ each, operated at a temperature of $10 \mathrm{mK} .{ }^{130} \mathrm{Te}$ decays exclusively by double beta decay processes and represents $34 \%$ of natural tellurium. The effective mass of CUORE will be $200 \mathrm{~kg}$ of ${ }^{130} \mathrm{Te}$ without enrichment. With 5 years of running at the nominal background levels of 0.01 counts $/ \mathrm{keV} / \mathrm{kg} /$ year and the typical energy resolution of $5 \mathrm{keV}$ FWHM, CUORE promises to be one of the most sensitive $0 \nu \beta \beta$ experiments in the next decade. The stated goal is the sensitivity of $T_{1 / 2}^{0 \nu}=2.1 \times 10^{26}$ years, which would correspond to the sensitivity to the effective neutrino mass of $45-70 \mathrm{meV}$, depending on the nuclear matrix elements. CUORE is currently under construction at the Gran Sasso Underground Laboratory (LNGS) in Italy, and is planned to start physics operations in 2013.

The detector techniques to be used in CUORE have been employed by the Cuoricino experiment at LNGS. Cuoricino operated between 2003-2008 with a $\mathrm{TeO}_{2}$ detector mass of $40.7 \mathrm{~kg}$. In five years of running with the present background level, Cuoricino gives an effective neutrino mass upper limit of about $300 \mathrm{meV}$. The final data analysis is in progress. The CUORE collaboration plans to use the Cuoricino cryostat for the operations of CUORE-0, a single-tower detector $1 / 19$ th the size of CUORE, constructed according to CUORE specifications. CUORE-0 should start running next year, before the CUORE detector is fully assembled, and due to the lower background levels, will overtake Cuoricino sensitivity in about a year of operations.

CUORE is a collaboration between the US and Italy. The Berkeley group is the largest US participant. LBNL is the lead laboratory, responsible for the project management, production of neutron transmutation-doped thermistors, user specification of the clean room facility, online and offline infrastructure, and data analysis. The current group includes senior staff scientists Freedman (NSD), Kolomensky and Kadel (PD), Beeman and Haller (MSD), and Smith (EH\&S). The group includes five postdocs (who share their time with other projects, e.g., KamLAND) and two graduate students. The Cuoricino group included 3 senior scientists, Norman (NSD, departed to LLNL), Xu (NSD), Haller, two postdocs, and one Ph.D. student. 


\subsubsection{High pressure Xenon detector R\&D2}

LBNL staff Nygren, Spieler (PD), and Goldschmidt (NSD) have been pursuing the development of a high pressure gas TPC (time projection chamber) with electroluminescence gain. This new technique may provide excellent energy resolution - just 3 times worse than Ge diode but 5 times better than liquid Xenon — and track imaging. Both energy resolution and track imaging are of key importance in searches for neutrinoless double beta decay, since they determine the extent to which backgrounds from gamma rays and 2-neutrino double beta decay can be reduced. The technique is expected to scale well to the 1 ton scale required for next generation searches in the $50 \mathrm{meV}$ effective mass region. The effort is being pursued in collaboration with staff at LLNL and Texas A\&M. Close ties exist also with the large NEXT collaboration in Spain pursuing similar research paths. LBNL is deeply involved in the development of the detector concept, calculations for detector optimization, and GEANT4 Monte Carlo simulations of the expected detector performance, and aims to contruct, optimize and characterize a test detector once funding permits.

LDRD funding for 2010 was requested through the physics division, at the level of $250 \mathrm{k} \$ /$ year for a period of 3 years. It is currently expected that the required energy resolution can be demonstrated with $1 \mathrm{~kg}$ of Xenon in a $0.25 \mathrm{~m}$ TPC about two years into the LDRD grant, and that design studies of a large scale experiment could be completed by the end of the third year. If successful, this detection technique could become the leading technique in future large scale experiments that search for neutrinoless double beta decay. In July 2009 DOE provided $250 \mathrm{k} \$(150 \mathrm{k} \$$ for LBNL, $100 \mathrm{k} \$$ for LLNL) through the Advanced Technology R\&D program for the engineering design of a $10 \mathrm{~kg}$ chamber and for the construction of a $1 \mathrm{~kg}$ prototype using an existing pressure vessel on loan from LLNL.

\subsection{Opportunities}

\section{Majorana}

LBNL has many core competencies and strengths that are essential to the success of Majorana. These include: HPGe detector, low-noise readout, and digitization expertise; personnel with experience on KamLAND, SNO, previous $0 \nu \beta \beta$ experiments, and GRETINA / Compton imaging experiments; proximity to and interaction with the DUSEL Project Team, UCB (where the management for DUSEL experiments will potentially reside), Al Smith's LBCF and related facilities for material assay, and PDSF for simulations and data analysis. Given the good overlap between various aspects of the MAJORANA experiment and LBNL core competencies, combined with LBNL's history of support and leadership in MAJORANA, LBNL is a natural candidate for lead laboratory of this experiment. LBNL has informed the MAJORANA collaboration if its interest in participating in this capacity.

Another significant MAJORANA task which LBNL is well-suited to handle is the detector task, including PPC detector procurement, readout electronics design, and detector integration. Kai Vetter already serves as the leader of this task, and LBNL personnel are already

\footnotetext{
${ }^{2}$ We thank Azriel Goldschmidt for help with writing this section.
} 
significantly involved in these activities. Other opportunities exist for significant LBNL contributions in digitization electronics, simulations and analysis, low-background counting, and management / DUSEL interface roles.

\section{CUORE}

CUORE will improve the sensitivity of the $0 \nu \beta \beta$ searches by two orders of magnitude. Should $0 \nu \beta \beta$ not be discovered at that level, further improvements in sensitivity will have to come from an increase in the detector mass, enrichment of the double beta decay isotopes, and a significant reduction of the background levels. Such an ambitious detector might need to be located deeper underground, in order to take full advantage of reduced environmental backgrounds and the larger detector mass. DUSEL at $7400 \mathrm{ft}$ level could be an ideal site for such an experiment.

A major weakness of any US-based proposal for a ton-scale bolometric double beta decay experiment is the fact that so far the US does not have a cryogenic installation dedicated to the development of the bolometric detectors for $0 \nu \beta \beta$ measurements. The LBNL group is planning to start a vigorous program of developing the next generation bolometric detectors suitable for searches for (or precision measurements of) neutrinoless double beta decays. Modest investments in the cryogenic low-background infrastructure would allow work on key elements of an important R\&D effort by developing readout techniques, testing new bolometric approaches, and to prepare the concept of a future experiment at DUSEL. The R\&D effort will focus on understanding the resolution limits of the bolometric detectors, on exploring the limitations and scalability of the isotopically-enriched double beta decay materials (including but not limited to enriched ${ }^{130} \mathrm{Te}$ ), and on developing the background suppression techniques. This long-range R\&D program will be geared toward a 1 ton scale, modular DBD detector to be available for deployment in DUSEL on the timescale of 2017 or later. The LBNL group has applied for LDRD funds.

\section{High pressure Xenon R\&D}

High pressure Xenon detection technology has the potential to transform future detectors to search for neutrinoless double beta decay and dark matter. LBNL is the natural place to pursue this R\&D effort, in view of the laboratory's core competences and the track record of the staff involved: the TPC, the proposed detector configuration, was invented by Nygren.

There is potential for interesting synergies with LZ3/LZ20. Dual phase Xenon dark matter and a gaseous Xenon TPC would both need to achieve long electron lifetime for drift and Xenon radiopurity. Some aspects of the shielding may also be common. An additional opportunity may arise for neutrinoless double beta decay: schemes are being discussed that may permit tagging of the Barium daughter following a decay in the high pressure Xenon gas. For instance, it may be possible to electromagnetically steer the $\mathrm{Ba}^{++}$ions to a location where an identification mechanism (still to be defined and developed) is activated. Barium tagging would eliminate all sources of background with the only exception of two-neutrino double beta decay, which is addressed by the excellent energy resolution. 


\section{Neutrino oscillations}

\subsection{Overview}

By now it is well established that neutrinos of different flavor can oscillate into one another, and therefore have non-zero masses. Apart from an anomaly reported by the LSND collaboration, which is not confirmed by MiniBoone, all data are consistent with the existence of three neutrino flavor eigenstates $\nu_{\alpha}(\alpha=e, \mu, \tau)$, which are related to the mass eigenstates $\nu_{i}(i=1,2,3)$ via $\left|\nu_{i}\right\rangle=\sum_{\alpha} U_{\alpha i}\left|\nu_{\alpha}\right\rangle$, where $U$ is the Maki-Nakagawa-Sakata (MNS) matrix,

$$
U=\left(\begin{array}{ccc}
U_{e 1} & U_{e 2} & U_{e 3} \\
U_{\mu 1} & U_{\mu 2} & U_{\mu 3} \\
U_{\tau 1} & U_{\tau 2} & U_{\tau 3}
\end{array}\right)=\left(\begin{array}{ccc}
1 & & \\
& c_{23} & s_{23} \\
& -s_{23} & c_{23}
\end{array}\right)\left(\begin{array}{ccc}
c_{13} & & s_{13} e^{-i \delta} \\
& 1 & \\
-s_{13} e^{i \delta} & & c_{13}
\end{array}\right)\left(\begin{array}{ccc}
c_{12} & s_{12} & \\
-s_{12} & c_{12} & \\
& & 1
\end{array}\right) .
$$

Here we have defined $s_{i j} \equiv \sin \theta_{i j}$ and $c_{i j} \equiv \cos \theta_{i j}$, and a non-vanishing complex phase, $\delta$, gives rise to $C P$ violation in the lepton sector. For Majorana neutrinos, the right-hand side of Eq. (2) is multiplied by $\operatorname{diag}\left(e^{i \alpha_{1} / 2}, e^{i \alpha_{2} / 2}, 1\right)$, where the additional $C P$ violating phases, $\alpha_{1,2}$, do not affect oscillation experiments, only $0 \nu \beta \beta$ decay.

The measurement of atmospheric neutrino oscillations determine $\Delta m_{23}^{2}=(1.9-3.0) \times$ $10^{-3} \mathrm{eV}^{2}$ and the mixing angle $\sin ^{2}\left(2 \theta_{23}\right)>0.92$ at $90 \%$ CL. KamLAND combined with measurements of solar neutrino oscillations determine $\Delta m_{12}^{2}=(7.59 \pm 0.21) \times 10^{-5} \mathrm{eV}^{2}$ and a mixing angle of $\sin ^{2}\left(2 \theta_{12}\right)=0.87_{-0.04}^{+0.03}$. The third mixing angle, $\theta_{13}$, has not been measured so far, and currently the experimental limit is $\sin ^{2}\left(2 \theta_{13}\right)<0.19$ at the $90 \% \mathrm{CL}$. The $C P$ violating phase, $\delta$, is completely unconstrained by the available data.

The goals in future neutrino experiments are the completion of the measurement of the neutrino mixing matrix, the study of $C P$ violation in the lepton sector, determining the sign of $\Delta m_{23}^{2}$ and looking for exotic effects. The importance of the sign of $\Delta m_{23}^{2}$ for $0 \nu \beta \beta$ was discussed already in Sec. 3.1. Measuring $\theta_{13}$ is interesting, because many theoretical models favor it to be not much smaller than the two observed mixing angles 3 (However, some scenarios, such as bimaximal mixing, imply $\theta_{13}=0$.) The value of $\theta_{13}$ is also important, because $C P$ violation in neutrino oscillation experiments is intrinsically a three (or more) generation phenomenon. Thus, any observable effect of the phase $\delta$ is multiplied by all three mixing angles, and $\theta_{13}$ in particular, for which only an upper bound exists so far. If $\sin ^{2} 2 \theta_{13} \gtrsim 0.01$ then $C P$ violation can be searched for using conventional beams, while otherwise searching for $C P$ violation will require the very intense and clean beams that a neutrino factory would make possible. The discovery of $C P$ violation in the lepton sector would make the leptogenesis explanation of the observed matter-antimatter asymmetry in the Universe quite plausible. Note, however, that the $C P$ violation responsible for leptogenesis in the decay of the very heavy "neutrinos" does not have a model independent relation to that observable in the oscillation of light neutrinos.

Several experiments under construction aim to improve the limit on $\sin ^{2}\left(2 \theta_{13}\right)$ down to a level around 0.01 . If $\sin ^{2}\left(2 \theta_{13}\right)$ has a larger value, then a future long baseline experiment will

\footnotetext{
${ }^{3}$ The parameters of the CKM quark mixing matrix are $\theta_{12} \approx 13^{\circ}, \theta_{23} \approx 2.4^{\circ}, \theta_{13} \approx 0.2^{\circ}$, and $\delta \approx \gamma \approx 68^{\circ}$. In the neutrino sector, $\sin ^{2}\left(2 \theta_{13}\right)<0.01$ would establish a factor of 10 hierarchy between $\theta_{12}$ and $\theta_{13}\left(<3^{\circ}\right)$.
} 
have sensitivity to $C P$ violation, while if $\sin ^{2}\left(2 \theta_{13}\right)$ is smaller, then such an experiment will be able to search for it down to the 0.005 level. A detector designed for observing neutrinos in a long baseline experiment also provides sensitivity to other interesting observables. For example, it would improve limits on the proton lifetime, with a sensitivity that probes many GUT models.

\subsection{Ongoing Berkeley activities}

\subsubsection{Sudbury Neutrino Observatory}

The Sudbury Neutrino Observatory (SNO) is a US, Canadian, British solar neutrino experiment located in INCO-Vale's Creighton \#9 Mine, near Sudbury, Ontario, Canada. To suppress cosmic ray backgrounds, SNO's $1 \mathrm{kt} \mathrm{D}_{2} \mathrm{O}$ target was positioned at the 6800 Level providing 6000 mwe shielding. Particular care was taken to assure the radio-purity of the detector components, including PMTs, acrylic vessel, and detector media. Queen's University was the lead institution in SNO. LBNL played the lead role in the PMT Support Structure, including design, engineering and installation. LBNL's Low Background Counting Facility provided essential low level assay capabilities to the collaboration. LBNL then played leading roles in the calibration, Monte Carlo and data analysis of the experiment. Lesko and Poon have chaired the collaboration's management committee. Poon served as the analysis coordinator for the salt and NCD phases.

The experiment was conducted in three phases: the first phase consisted of a pure $\mathrm{D}_{2} \mathrm{O}$ target, the second phase introduced $2 \mathrm{t}$ of $\mathrm{NaCl}$ to the detector to enhance neutron detection and analysis, the third phase introduced discrete ${ }^{3} \mathrm{He}$ detectors into the $\mathrm{D}_{2} \mathrm{O}$ to pursue an alternative neutron detection scheme. The experiment has stopped data collection, the $\mathrm{D}_{2} \mathrm{O}$ returned to AECL, and the final analysis efforts are underway.

The main focus for SNO was to resolve the solar neutrino problem. By observing flavor changing neutrino oscillations SNO resolved this 40-year-old problem. SNO demonstrated neutrino oscillations by measuring the appearance of muon and tau neutrino flavors in conjunction with the disappearance of electron neutrino flux. Exclusively and inclusively with other experiments, SNO measured $\theta_{12}$ and $\Delta m_{12}^{2}$. SNO's measurement provides the most precise determination of $\theta_{12}$, and determines that this mixing angle is not maximal.

LBNL's efforts in SNO began in 1989. Underground construction was initiated in 1994 and was completed in 1998. Data collection was conducted between 1999 and 2006, roughly divided equally between the three phases, with the Neutral Current Detectors requiring nearly two years to install. SNO's primary physics analyses are anticipated to conclude in 2010. LBNL's involvement was from the Nuclear Science Division and Engineering Division and included NSD senior staff Lesko, Norman and Stokstad, staff scientists Chan and Poon, and typically two to four post-docs and one to two GSRAs during any given year. Marino was awarded the APS Tanaka Prize for her 2004 dissertation on SNO's salt-phase analysis.

LBNL's hardware contributions to the SNO experiment included the PMT support structure design, engineering and installation as well as the design and construction of several calibration devices. At the height of SNO's engineering effort, LBNL provided four Me- 
chanical Engineers, two designers, and three technicians, who were primarily charged with underground installation. LBNL provided unique low background counting expertise and facilities to assure SNO's background requirements. Following construction LBNL's group provided major manpower to SNO's Monte Carlo and analysis efforts, including NERSC computing resources and data storage.

Through our involvement in SNO, LBNL developed core competencies in ultrapure water Cherenkov detector design and operation, ultralow background materials and assays, calibration devices, establishing clean room conditions underground, and data analysis and Monte Carlo techniques. These competencies are very relevant to future detector efforts including many discussed in this report.

\subsubsection{KamLAND}

KamLAND is a US-Japanese experiment involving more than ten US Universities and National Laboratories. The major Japanese institute is the University of Tohoku but three smaller Japanese universities have been involved during the course of the experiment. UC Berkeley and LBNL are the principal US institutes. The DOE funding for KamLAND through Berkeley as the lead laboratory started in 1998 as a joint DOE Nuclear and High Energy Physics initiative. Both Physics and Nuclear Science Divisions have participated in KamLAND. Five Physics Staff members and five Nuclear Science Staff members have been involved during the course of the project. Presently Staff members Steiner, Kadel and Murayama from Physics and Freedman and Fujikawa from Nuclear Science are actively involved in KamLAND. Three Ph.D. students and twelve Berkeley postdoctoral researchers have worked on the project. Two have gone on to postdoctoral positions at MIT and Caltech. Presently there is one graduate student and four postdoctoral researchers from the Nuclear Science Division and two more from campus. Berkeley provided major hardware components of KamLAND including the data taking electronics, electronics infrastructure and calibrations devices. The US data analysis was centered at Berkeley using the PDSF/NERSC facility. Berkeley provided the US project management during construction.

KamLAND is a long baseline neutrino oscillation experiment located in Kamioka Japan. The primary purpose is a study of the neutrino flux from Japanese reactors with a 1000-ton liquid scintillation detector. KamLAND has established the Large Mixing Angle solution to the Solar-neutrino problem and it provides by a significant margin the most precise measurement of a neutrino mass splitting. KamLAND gives us the most conclusive and direct evidence of neutrino oscillation. It gave us the first evidence of neutrino emission from the Earth, establishing geoneutrinos as a unique tool for geophysics. KamLAND is presently reducing the radioactive backgrounds for measurements of low energy solar neutrinos. A measurement of the high-energy solar neutrino flux will be published this year. The purification of KamLAND scintillator is working well and the quality of the data from KamLAND is constantly improving. It is expected that measurements of low energy solar neutrinos will begin soon. KamLAND is considered one of the most successful neutrino oscillation experiments worldwide.

Berkeley remains the center of KamLAND activity in the US. Recently the KamLAND 
collaboration meeting was held in Berkeley. US analysis activities continue to be led by Berkeley. A weekly analysis meeting and a biweekly purification meeting are held at Berkeley. Three new papers on KamLAND physics are in process. We expect new graduate students to join now that the new phase of solar neutrino research is beginning.

\subsubsection{Daya Bay田}

The Daya Bay Collaboration aims to determine the neutrino mixing angle $\theta_{13}$ by comparing the flux and energy spectrum of the electron antineutrinos produced by the Daya Bay Nuclear Power Facility in China at two different baselines using $0.1 \%$ Gd-loaded liquid scintillator. The ultimate sensitivity in $\sin ^{2}\left(2 \theta_{13}\right)$ at $90 \%$ CL is 0.008 , while the $3 \sigma$ and $5 \sigma$ discovery limits are 0.0146 and 0.0244 , respectively 5 The outcome of the experiment is important for the future program, since the smaller $\theta_{13}$ is, the harder it will be to distinguish normal and inverted hierarchy and to look for $C P$ violation in neutrino oscillation. If $\sin ^{2}\left(2 \theta_{13}\right)$ is significantly larger than 0.01 , it will be feasible to study $C P$ violation in neutrino oscillation using conventional (anti-)neutrino beams.

The collaboration consists of about 230 scientific and technical staff from 37 institutions world wide. The experiment is funded at the level of RMB $\$ 155 \mathrm{M}$ (provided by China for civil construction and detector), US\$ 34M (by DOE for the detector), and HK\$ $10 \mathrm{M}$ (Hong Kong). In addition, Taiwan is providing NT $\$ 40 \mathrm{M}$ over a period spanning 2008-2012, and the Czech Republic contributed US\$ 0.5M. The experiment is currently under construction. It is anticipated that the two initial detectors will be commissioned in the first near hall underground in Summer 2010. Data taking with all eight detectors in three underground halls is expected to begin in late 2011 and will last for three years.

The Berkeley Staff include Luk (co-spokesperson), Edwards (project manager), Lin, Steiner, two postdocs (Liu, Zhong), as well as computing (Tull, Patton, Lee) and engineering (Barry, Stuart, Virostek, Joseph, Zimmerman) support. LBNL holds the US scientific and project leadership, and provides about 2600 photomultiplier tubes with the associated mechanical structure and HV system, as well as an apparatus for measuring the attenuation length of liquids used in the antineutrino detectors onsite. Further LBNL contributions include low-background counting expertise, and software development.

\subsubsection{Long baseline neutrino experiment}

The accelerator driven goals of the DUSEL large cavity detectors are:

- Measure $C P$ violation in neutrino sector;

- Determine the neutrino mass hierarchy (normal or inverted);

- Measure the value of $\sin ^{2} 2 \theta_{13}$.

The non-accelerator physics goals are:

- Detect proton decay;

\footnotetext{
${ }^{4}$ We thank Kam-Biu Luk for help with writing this section.

${ }^{5}$ The Double Chooz experiment expects to measure $\theta_{13}$ with $3 \sigma$ significance if $\sin ^{2}\left(2 \theta_{13}\right)>0.05$, or to exclude it at $90 \%$ CL down to 0.03 for $\Delta m^{2}=2.5 \times 10^{-3} \mathrm{eV}^{2}$ in 3 years of data taking with both detectors.
} 
- Supernova searches;

- Detect diffuse neutrinos from past supernova;

- High precision measurement of solar neutrinos, etc.

For accelerator driven physics no detector technology or physics process requires a depth greater than 1000 meter water equivalent (mwe). This follows from the short live-time of the experiment for accelerator physics: only about $100 \mathrm{sec} / \mathrm{yr}\left(10^{7} \mathrm{pulses} / \mathrm{yr} \times 10 \mu \mathrm{sec} / \mathrm{pulse}\right)$. The depth of the detector is defined largely by the proton decay search requirements. Sufficient depth is needed to suppress background from neutral kaons produced in the surrounding rock, which enter the fiducial volume undetected and their subsequent decay appears to mimic the proton decay $p \rightarrow K^{+} \bar{\nu}$. An overburden of at least 3000 mwe is necessary to suppress this background and still retain $90 \%$ of the detector fiducial volume. No process of interest requires a depth greater than the $4850 \mathrm{ft}$ of the DUSEL mid-level campus.

In January, the NSF conducted a first year review of the DUSEL cooperative agreement between NSF and UC Berkeley. Kadel presented the physics and depth arguments to the review committee and placed the LBNE experiment in the context of its international competition, discussed the coordination between DUSEL facilities and LBNE, including the definition of cavity requirements for both technologies and the cost and schedule for geotechnical investigations of the site. This presentation emphasized the depth and size requirements for a large cavity detector, indicating the need for a minimum of $300 \mathrm{kT}$ fiducial volume for a water Cherenkov detector, contained in three or fewer cavities of $100 \mathrm{kT}$ fiducial volume each. A liquid argon detector of fiducial mass greater or equal to $50 \mathrm{kT}$ is an excellent complement to the water Chrenkov detector because of its different sensitivity to $\nu$ vs. $\bar{\nu}$ events from astrophysical sources, potentially better pattern recognition capabilities, and higher efficiency for the $p \rightarrow K^{+} \bar{\nu}$ decay, should supersymmetry be seen at the LHC. This technology is largely in its infancy, although a robust R\&D program exists (e.g., ArgoNeuT and microBoone at FNAL). The overall comments of the review panel large cavity detectors can be summarized as follows:

- Science is well motivated;

- Integration issues understood in terms of depth requirements, size and interface between the two possible experimental techniques and the facility.

- Significant progress; List of integration issues appropriate for project status;

- Congratulations to DUSEL team and collaboration for identifying science requirements for experiment and facility infrastructure.

This is a new program for the LBNL Physics Division, only about one year old. There is currently one person (Kadel) involved in the PD. He holds the DUSEL position of Deputy Director for Large Cavity Detectors and is a member of the LBNE experiment. Kadel serves on the LBNE executive committee, collaboration council and BNL-FNAL-LBNL (DUSEL) Program Management Group.

\subsection{Opportunities}

The SNO, KamLAND, and Daya Bay experiments have their plans in place, and will all finish before LBNE has data. The research plan for LBNE depends on the R\&D funding 
from the NSF S4 for DUSEL and the level of DOE funding for the LBNE, neither of which has yet been determined. LBNL has proposed:

1) Electrical engineering resources to provide a cost/benefit and mean time between failure analysis of front end electronics for the water Cherenkov detectors located above the water level compared to electronics located underwater directly near the PMTs (DOE funded, submitted to LBNE Project office).

2) Continue to provide coordination between the DUSEL facility and the large cavity experiment (NSF).

3) After additional reflection and discussion within the DUSEL project and with the scientific collaboration, it is apparent that the cavity liner and drain system is too closely linked to the cavity excavation to introduce a group external to the cavity design group. These systems present major interfaces that must be extremely well coordinated. We propose to move the conceptual design work and associated R\&D closer to the DUSEL cavity design team, and include additional scope for LBNL's participation in the LBNE program. This co-locates the cavity excavation and liner design efforts and promotes the coordination and integration of these efforts (DOE, submitted to LBNE Project office).

As described, currently there is one member of the Physics Division senior staff working on the LBNE collaboration. A modest increase in FTEs would be needed to pursue this opportunity in the coming years. In the past PD and NSD have successfully used a model in which postdocs participate about equal time on a future project and on an existing experiment on which they can analyze data. This model could also be used for LBNE.

\section{Dark matter searches}

\subsection{Overview}

The identity of dark matter in the universe is a central question in particle physics and cosmology. While the existence of dark matter is well established by cosmological observations on a wide range of scales from sub-galactic to horizon-size, its nature is largely unknown. The experimentally allowed range for its mass and cross section spans many orders of magnitude. We know that dark matter cannot be a particle in the standard model in order not to contradict successful theories of big-bang nucleosynthesis and structure formation. Knowing more about the properties of dark matter will, therefore, have strong and broad implications on fundamental particle physics as well as astrophysics.

Why do we think that dark matter physics will have significant progress in the "near" future, in particular on the DUSEL time scale? While we do not know much about the particle physics nature of dark matter, we know some about its gravitational nature. In particular, we know the precise abundance of dark matter in the current universe, especially due to WMAP measurements. Suppose that dark matter is in thermal equilibrium with the hot plasma in the very early universe, which is a plausible assumption. The current relic density of dark matter is then determined by its self-annihilation cross section. The observed abundance of dark matter implies that the necessarily cross section is of order $1 / \mathrm{TeV}^{2}$. This is a very interesting coincidence - the $\mathrm{TeV}$ scale is the most important 
scale in current particle physics, where we expect to see new physics beyond the standard model. This coincidence provides a strong motivation that dark matter may be a weakly interacting massive particle (WIMP), which may be associated with TeV-scale new physics related to electroweak symmetry breaking. In fact, many new physics scenarios beyond the standard model, such as supersymmetry and extra dimensions, do provide viable candidates for TeV-scale dark matter.

It is this association of dark matter to weak-scale physics that makes future progress very promising. The dark matter having weak-scale cross sections implies that we may see it directly, through its recoil off nuclei in experimental apparatus, or indirectly, though detecting products of its annihilation in the Earth, Sun, and galaxy. The sensitivity of current experiments have started to reach the expected regions for the WIMP mass and cross sections. Dark matter may even be produced at the LHC, depending on models. All these experiments are complementary in many respects, and it is expected that they can reveal the nature of dark matter if the idea of weakly interacting dark matter is correct. DUSEL provides a very important opportunity for direct detection experiments because of its low background environment. There are several experiments proposed, which can probe various aspects of dark matter — mass, spin-independent cross section, spin-dependent cross section, directional information, dependence of cross sections on target nuclei, and so on - with much better sensitivities than earlier experiments. It is an extremely important endeavor to figure out properties of dark matter, or to discover it if it is not discovered by then. For example, experiments using Germanium and cryogenic Xenon discussed later may probe a significant portion of the parameter space for naive weakly interacting dark matter. The result of such experiments, even if negative, will have tremendous impact on the theory of dark matter.

\section{$5.2 \quad$ Ongoing Berkeley activities}

\subsubsection{CDMS / Super-CDMS / GEODM6}

Low temperature Germanium detectors represent one of the leading approaches for the direct search for WIMPs, which may constitute the dark matter in the universe and a signature of the new physics needed at the electroweak scale. The simultaneous detection of phonons and ionization is, so far, the only demonstrated technology that is background free at the current level of sensitivity. As a consequence, CDMS II is currently the most sensitive experiment at large masses. New results with a sensitivity increase of a factor of three are expected in the coming months. With DOE and NSF support, the team of about 40 physicists is currently extrapolating from the current target mass of $4 \mathrm{~kg}$ to $15 \mathrm{~kg}$ (3" diameter, 1 " thickness) to be run at Soudan. The first phase of this program is funded and the decision for the second phase will be taken in the Summer 2009.

By going to larger detectors (6" diameter and 2" thickness, 5kg/detector) and using multiplexing (32-128 phonons sensors/detectors), one can envision an extrapolation of the target mass to the $150 \mathrm{~kg}$ scale for an experiment at SNOLAB starting in 2012 and to ton

\footnotetext{
${ }^{6}$ We thank Bernard Sadoulet for help with writing this section.
} 
scale at DUSEL (7400 feet, 2017), while maintaining zero background. These target masses are necessary both to push down the sensitivity, in case no signal is seen and to increase the statistics if a signal is seen. An S4 proposal for GEODM (Germanium Observatory of Dark Matter DUSEL) has been submitted to NSF, and a complementary proposal is being prepared for DOE.

LBNL has played an important role in the startup of CDMS. At present, the Berkeley campus group around Bernard Sadoulet continues to be one of the main CDMS groups, strongly involved in long-term technology development, detector testing and characterization and in analysis.

\subsubsection{LUX / LZ3 / LZ207}

LUX is a $350 \mathrm{~kg}$ monolithic dual phase (liquid-gas) Xe Time Projection Chamber (TPC) that has the capability to detect WIMPs through elastic scattering off of Xe nuclei, a technique previously exploited by the XENON10, ZEPLIN-II, and ZEPLIN-III experiments. With a one year exposure, LUX can be sensitive to cross sections of the order of $10^{-45} \mathrm{~cm}^{2}$ for WIMPs with a mass of $100 \mathrm{GeV} / \mathrm{c}^{2}$. To achieve this sensitivity, the electron recoil background rate has to be less than $8.3 \times 10^{-4}$ evts $/ \mathrm{keV} / \mathrm{kg} /$ day, requiring the $\mathrm{U} / \mathrm{Th}$ levels to be limited at the tens of ppt level in the cryostat and surrounding materials. LUX is funded at the level of $\$ 2.5 \mathrm{M}$ from NSF and DOE (HEP), plus an equivalent amount from the university partners. Sanford Lab is providing $\$ 5.5 \mathrm{M}$ in laboratory support for the active water shield, infrastructure, and related items. The LUX experiment is scheduled to begin construction in 2009, with data taking starting in 2010. However, this schedule is highly dependent upon the availability of the Davis Cavern at the Sanford Lab's $4850 \mathrm{ft}$ level and it is probably optimistic. At the present time, the LUX collaboration consists of approximately 50 scientists, including three from Berkeley (Lesko, Chan, and Fujikawa). The roles of the Berkeley scientists involve interfacing with the Sanford Lab and with the low background counting of materials used in the experiment, and the level of involvement is relatively minor.

To first order, LZ3/LZ20 is the LUX detector scaled up to larger Xe mass, 3 and 20 tons, respectively. With the larger target mass and improved signal-to-noise - from the self-shielding effects of a larger detector — LZ3 can be sensitive to cross sections of the order of $10^{-46} \mathrm{~cm}^{2}$ for WIMPs with a mass of $100 \mathrm{GeV} / \mathrm{c}^{2}$ and LZ20 can be sensitive down to $10^{-48} \mathrm{~cm}^{2}$. The LZ3 detector is planned to be installed in the Davis Cavern at the Sanford Lab's $4850 \mathrm{ft}$ level and it will use the same water shield as LUX. The possibility of employing the LZ3/LZ20 detectors to search for ${ }^{136}$ Xe neutrinoless double beta decay and to measure solar neutrino fluxes is currently being explored. The LZ20 collaboration, which is a merger of the LUX and ZEPLIN collaborations, submitted an S4 proposal to NSF, with the plan of taking data in 2012. At the present time, three Berkeley scientists (Fujikawa, Jacobsen, and Siegrist) are LZ20 collaborators, and a Berkeley engineer (von der Lippe) is the project manager. As with LUX, the current level of participation by the Berkeley scientists is very low, well below 0.1 FTE.

\footnotetext{
${ }^{7}$ We thank Brian Fujikawa for help with writing this section.
} 


\subsection{Opportunities}

\section{CDMS / Super-CDMS / GEODM}

There are many areas where LBNL could get involved again, in particular to help with its Germanium expertise in the development of large diameter crystals (with zero dislocations) and improvement of contacts. Although the low temperature behavior is different, significant synergies exist with Majorana and if the growth of large crystals were feasible, this could have an impact of Homeland Security / non-proliferation applications. Another area where LBNL core competences could be applied is RF multiplexing with fast ADC, DAC and FPGA processing. This could involve interested scientist in PD (in addition to Sadoulet) and NSD (in particular, Majorana physicists), and Engineering. Participation in GEODM would naturally lead to involvement of the scientists in the pre-DUSEL CDMS program at Soudan and SNOLAB. SLAC has decided to get involved in CDMS, and the collaboration of LBNL with SLAC, Fermilab and the university groups would be very powerful.

\section{LUX / LZ3 / LZ20}

During a visit to LBNL, Akerib, Gaitskell, and Shutt have expressed their interest in having a larger participation by Berkeley in LUX/LZ3/LZ20, including a substantial engineering role. Several members of NSD have also expressed interest in applying their expertise in measuring rare events, obtained from the SNO, KamLAND, and Majorana neutrino experiments, to Dark Matter experiments such as LUX/LZ3/LZ20. In addition, Goldschmidt who is currently doing R\&D on high pressure Xe TPC detector, has expressed interest in working synergistically with LUX/LZ3/LZ20 on Xe handing and purification. Dark matter searches have traditionally been supported by DOE-HEP. It may be shown that LZ3/LZ20 could contribute significantly to the search for neutrinoless double beta decay and/or to precision measurements of solar neutrino fluxes. This may provide a better fit with efforts traditionally supported by DOE-NP. Unless support by DOE-NP can be obtained, significant participation in LUX/LZ3/LZ20 may be limited to the Physics and Engineering Divisions.

\section{Recommendations}

The committee reviewed experiments that may become part of the integrated suite of DUSEL experiments or that may lead to experiments sited in DUSEL on a longer timescale. Experiments in the areas of nuclear astrophysics, neutrinoless double beta decay, long baseline neutrinos and proton decay, and dark matter searches were described in a series of Division seminars and Research Progress Meetings, listed in the appendix to this report. Compelling science was presented in each of these areas.

The Berkeley community is already deeply involved in a number of experiments and/or proposals that will be relevant to science at DUSEL (see Table 1). The committee has identified no compelling reasons, at this time, to devote resources at Berkeley to potential future experiments (and related R\&D) beyond those activities that already have significant 
Berkeley involvement. The scientific involvement in some of the projects, however, should be strengthened.

Cooperation and collaboration among the Physics and Nuclear Science Divisions and UC Berkeley, with strong technical support from the Engineering Division (and in a few cases other Divisions), is a central feature of the current broad program. The Berkeley community has a unique role in the development of the DUSEL facility, which itself will require substantial scientific and technical (engineering) leadership from LBNL. Future experiments at DUSEL will present new challenges in technical scale and complexity, cost and duration from conception to initiation of data analysis. LBNL's present scientific involvement in the proposed integrated suite of experiments is not commensurate with its role in the DUSEL facility. There is a significant risk that a Berkeley leadership role in science at DUSEL will not be possible unless additional personnel are found to maintain scientific and technical leadership in DUSEL experiments. It is essential that the cooperation and joint planning for DUSEL experiments among the PD, NSD, ED, and UCB be strengthened to meet these challenges. We recommend that the leadership of the PD, NSD, ED, and the UCB Physics Department should establish the means for personnel planning to provide scientific and technical leadership from now through the completion of the first major DUSEL experiments in the 2020 decade.

Our specific recommendations below focus on experiments which we think meet the following conditions:

1. Compelling science: fundamental to nuclear or particle physics.

2. Appropriate for LBNL: we can make a unique contribution, whether in scale, technology, experience or resources, consistent with the long range plans of the funding agencies.

3. Local interest and expertise.

4. Scientific and technical feasibility: while there is unavoidable uncertainty in long-term R\&D projects, there are no clear show-stoppers.

5. Within the realm of possibility: reasonable chance for funding, attracting local resources, utilizing interdivisional / UCB collaboration.

\section{Specific recommendations:}

- DIANA: LBNL holds the lead role in the accelerator development of the DIANA proposal. We recommend that, as DIANA progresses through review, the existing involvement in the accelerator becomes paired with commensurate scientific involvement if resources can be found. We recommend that, if additional resources cannot be found as DIANA progresses through review, the involvement in DIANA be revisited in the context of other commitments.

- Majorana: LBNL has a significant scientific investment and involvement in the Majorana Demonstrator and the Majorana experiment. A plan that leads to a sustainable leadership role for LBNL in Majorana on the timescale of the 1 ton experiment needs to be detailed. Re-establishing senior leadership to the LBNL effort is likely to be a necessary element of such a plan. 
- Long Baseline Collaboration: We recommend to modestly expand the current involvement in a multi-100 kT Water Cherenkov detector if funds can be attracted through DUSEL, the LBNE project, or other sources. This could provide a basis for increased future scientific involvement, which should be revisited when the results of current experiments determining $\theta_{13}$ are known.

- Dark Matter: Dark Matter experiments, in particular LUX/LZ20 and CDMS/GEODM, offer compelling science and excellent opportunities for LBNL leadership roles and technical contributions. The committee feels that not having a significant scientific role in a Dark Matter experiment would be a missed opportunity. The committee also believes that LBNL can have a significant scientific impact on at most one of these projects, and that credible scientific participation will likely require new resources. We recommend to identify the resources that would be needed for a credible scientific participation in either LZ20 or GEODM. A decision for either of these experiments should be driven by the scientific leadership of the LBNL effort.

- RED: We strongly encourage R\&D. In particular, there are ongoing R\&D efforts in cryogenic bolometry (related to CUORE) and high pressure Xenon TPCs (related to neutrinoless double beta decay, and possibly dark matter experiments). If successful, these R\&D efforts may provide the basis for one or more future generations of DUSEL experiments. 


\section{Appendices}

\section{A Low Background Counting and Radiological Assay}

Many underground experiments share a common requirement that detector components be manufactured from materials with low levels of radioactive species including $\mathrm{U}$ and Th series, $\mathrm{K}$, and other common long-lived species.

For DUSEL's integrated suite of experiments the need to control radioactive backgrounds is an extremely high priority for the neutrinoless double beta decay experiments, solar neutrino experiments, dark matter experiments, and nuclear astrophysics experiments.

LBNL's low background counting facility (LBCF) includes specialized detector arrays at the Building 72 facility and in the well-shielded Oroville underground facility. These facilities have operated for nearly thirty years, providing assay services to the LBNL-UCSB Ge double beta decay, SNO, KamLAND, MAJORANA, Cuoricino, CUORE, Daya Bay, KATRIN, LUX, Double Chooz and other experimental collaborations.

The LBCF employs direct and neutron-activated $\gamma$-ray counting techniques. With direct counting sensitivities for $[\mathrm{U}, \mathrm{Th}]$ to $\sim 1 \mathrm{mBq} / \mathrm{kg}$ are obtained. Combining neutron activation additionally enhances the sensitivity levels to $10^{-15} \mathrm{~g} / \mathrm{g}$.

\section{B DUSEL Working Group}

\section{Charge to the committee}

"In the context of the scientific opportunities in high energy and nuclear physics presented by DUSEL, the Committee is asked to assess the present LBNL participation in its initial suite of experiments, and to propose a roadmap for our future participation in the facility. Proposals for experimental development have been submitted to the NSF (S4) in the following broad areas: Long Baseline Neutrinos, Proton Decay, Neutrinoless Double Beta Decay, Dark Matter Searches, Nuclear Astrophysics Experiments and Low Background Counting. For each topic, the committee should identify the scientific opportunity, and summarize the scale of current $L B N L$ participation, the impact that $L B N L$ is likely to have on experiments at the present level of effort, the value of additional manpower, and opportunities for synergistic Detector RED activities.

Since both NP and HEP funding is anticipated for DUSEL experiments, the Committee should comment on the match of the proposed experiments to the HEP P5 report and the NP long range plan.

DUSEL presents many opportunities, but the roadmap should be presented in the context of long-term commitments to other high priority programs such as JDEM, LHC, RHIC and existing neutrino efforts such as Daya Bay, KamLAND and CUORE." 


\section{Working group members}

Christian Bauer (PD)

Jason Detwiler (NSD)

Stuart Freedman (NSD, UCB)

Murdock Gilchriese (PD)

Richard Kadel (PD)

Volker Koch (NSD)

Yury Kolomensky (PD, UCB)

Kevin Lesko (NSD)
Zoltan Ligeti (PD, co-chair)

Henrik von der Lippe (ENG)

Steve Marks (ENG)

Yasunori Nomura (PD, UCB)

David Plate (ENG)

Natalie Roe (PD)

Ernst Sichtermann (NSD, co-chair)

\section{Working group meetings}

\begin{tabular}{lll}
\hline \hline \multicolumn{1}{c}{ Topic } & Presenter & Date \\
\hline \hline Dark Matter: Current and Future & Y. Nomura & Feb 5 \\
The DRIFT detector & D. Loomba & Feb 6 \\
Discussion of the Charge & J. Siegrist \& J. Symons & Feb 10 \\
LUX, LZ20 and the race to detect WIMP dark matter & T. Shutt & Feb 10 \\
Directional Dark Matter Search & G. Sciolla & Feb 12 \\
Some Science Motivations for DUSEL & W. Haxton & Feb 24 \\
Germanium Observatory for Dark Matter at DUSEL (GEODM) $)$ & B. Sadoulet & Feb 26 \\
MAX: Multi-ton Argon and Xenon TPCs & C. Galbiati & Mar 3 \\
Preparations for writing the report & Z. Ligeti \& E. Sichtermann & Mar 13 \\
The US LAr TPC Program Leading to DUSEL & B. Fleming & Mar 17 \\
EXO Status and Perspectives & G. Gratta & Mar 20 \\
DIANA & D. Leitner \& P. Vetter & Mar 24 \\
Development of a High Pressure Xenon Imager & A. Goldschmidt & Mar 25 \\
Present Status of COUPP & J. Collar & Mar 26 \\
Large Cavity Detectors at DUSEL & R. Kadel & Mar 31 \\
The Majorana Project & S. Elliott & Apr 7 \\
Analysis and discussions for the report & all & Apr 10, 17, 24 \\
& & May 1, 5, 8, 15 \\
\hline \hline
\end{tabular}

\section{Acknowledgments}

This work was supported in part by the U.S. Department of Energy, Office of Science, Offices of High Energy Physics and Nuclear Physics, under contract DE-AC02-05CH11231. 